\title{
The impact of Silymarin on improvement of platelet abnormalities in patients with severe preeclampsia
}

\author{
Fahimeh Kaveh Baghbahadorani ${ }^{1}$, Sepideh Miraj $^{2}$
}

${ }^{1}$ Resident of Gynecology, Faculty of Medicine, Shahrekord University of Medical Sciences, Shahrekord, Iran

${ }^{2}$ M.D., Gynecologist, Fellowship of Infertility, Assistant Professor, Faculty of Medicine, Shahrekord University of Medical Sciences, Shahrekord, Iran

\section{Type of article: Original}

\begin{abstract}
Background: Preeclampsia is a pregnancy-specific disorder that is associated with an increase in blood pressure and proteinuria; in severe cases, it can cause platelet abnormalities. Silymarin is the extract of Silybum marianum, which is recognized as a safe antioxidant drug.

Objective: To determine the impact of Silymarin on the improvement of severe preeclampsia in 60 patients with severe preeclampsia.

Methods: In this double-blind clinical trial study, This study included 60 patients whose pregnancies were terminated because of severe preeclampsia and who were referred to Hajar Hospital in Shahrekord, Iran, from April 2014 to September 2015. The patients were divided randomly into two groups, i.e., a group of 30 patients and a control group of 30 patients. In addition to the current treatments for preeclampsia, The members of the study group were administered $70 \mathrm{mg}$ of Silymarin at three hours and 24 hours after the termination of their pregnancies. The control group received a placebo at the same times. Platelet count tests were compared at the baseline and at 12, 36, and 60 hours post-measurements in the two groups by SPSS software, version 22, by the ANOVA test, and by the independent-samples t-test.

Results: At the baseline, the two groups were not significantly different in terms of various criteria, such as age, BMI, and platelet counts. There were no significant differences between the two groups regarding the number of platelets at 12,36 , and $60 \mathrm{~h}$ after their pregnancies were ended $(\mathrm{p}>0.01)$.

Conclusions: The results of this study indicated that, although oxidative factors are involved in the incidence of complications of preeclampsia, e.g., thrombocytopenia, merely using an oxidative agent does not alleviate this effect. This indicated that other factors likely are involved in the pathogenesis of this disease. Additional studies are needed to prove the beneficial effects of this drug in the treatment of preeclampsia.

Clinical trial registration: The trial was registered at the Iranian Registry of Clinical Trials (http://www.irct.ir) with the Irct ID: IRCT201509042388/N1.

Funding: Shahrekord University of Medical Sciences supported this research (project no. 2006).

Keywords: Preeclampsia, Silymarin, Platelet count
\end{abstract}

\section{Introduction}

Preeclampsia is a multifactorial disease, and it is a major cause of maternal and fetal morbidity and mortality. Preeclampsia is defined as a sudden onset of hypertension, proteinuria, or organ failure after the 20th week of pregnancy in women who previously had normal blood pressure. Clinical manifestation of preeclampsia may appear at any time during the second half of the pregnancy or during the first few days after delivery. Patients who have severe preeclampsia may have various disorders, such as pulmonary edema, cerebral hemorrhage, convulsions, impaired hepatic abnormalities, renal impairment, and even death. In addition, newborns can experience complications, such as preterm delivery, stillbirth, neonatal growth restriction, and neonatal admission to the intensive care center (1). The features of severe preeclampsia include systolic blood pressure greater than or equal to

\section{Corresponding author:}

Assiastant Professor Dr. Sepideh Miraj, Faculty of Medicine, Shahrekord University of Medical Sciences, Shahrekord, Iran. Email: miraj.sepideh@gmail.com

Received: January 02, 2016, Accepted: May 06, 2016, Published: May 2016

iThenticate screening: May 02, 2016, English editing: May 12, 2016, Quality control: May 14, 2016

(C) 2016 The Authors. This is an open access article under the terms of the Creative Commons Attribution-NonCommercialNoDerivs License, which permits use and distribution in any medium, provided the original work is properly cited, the use is non-commercial and no modifications or adaptations are made. 
$160 \mathrm{~mm} \mathrm{Hg}$, diastolic blood pressure greater than or equal to $110 \mathrm{~mm} \mathrm{Hg}$, severe and persistent headache, visual disturbances, upper abdominal pain, thrombocytopenia, microangiopathic hemodialysis, and increases in creatinine and liver enzymes (2). The main risk of preeclampsia for the fetus is a decrease in uterine placental blood flow, and the only way to reduce the symptoms of preeclampsia effectively is delivery of the baby. Perinatal mortality occurs in many patients and it is due to lack of maturity of the fetus that results from premature labor. Preeclampsia is the cause of about $15 \%$ of preterm deliveries (3). Risk factors for preeclampsia include pregnancy at an early or an old age, first pregnancy, history of chronic hypertension, obesity, multifetal delivery, and a history of preeclampsia in a previous pregnancy (4). The exact pathogenesis of preeclampsia is not yet clear, but several hypotheses has been proposed, including the impact of immunological factors, coagulation disorders, lack of adaptation of the mother regarding cardiovascular changes or inflammatory compliance during pregnancy, genetic factors, nutritional factors, and increased production of free radicals of oxygen, and, consequently, the plasma lipid peroxidation that can cause endothelial damage and placental vascularization $(4,5)$.

Several studies have reported an imbalance between the antioxidants and lipid peroxidation in preeclampsia. In this study, the impact of silymarin, an herb with antioxidant properties, was investigated to determine its effectiveness in improving severe preeclampsia. Silymarin is the extract of the Silybum marianum herb. Silymarin is a medicinal plant and is the extract of Silybum marianum. Silybum marianum (Silybum) was considered as a sacred drug in ancient times and as a traditional medical treatment, and it has been used as a medicinal plant for many years. In the first century AD, the Romanian biologist, Pliny, stated that the milky extract of this plant was useful for inducing the secretion of bile. In 1968, scientists separated three specific molecules from this plant that protect the liver, i.e., Silibinin, Silydiannin, and Silychristin, and they now collectively are called silymarin. The therapeutic use of this plant dates back more than 2000 years, and in ancient Greek sources, it was referred to as a liver protective agent (5, 6). Several recent studies have examined the effectiveness of silymarin as antioxidant drug, and they found that it had beneficial effects in most patients and had no significant side effects. In this study, we determined the impact of silymarin on different symptoms of severe preeclampsia in pregnant women whose pregnancies were terminated due to severe preeclampsia.

\section{Material and methods}

\subsection{Trial design and participants}

This study was a randomized clinical trial that was conducted from April 2014 to September 2015. This clinical trial was conducted in an Iranian governmental educational hospital (Hajar Hospital). This hospital is affiliated with Shahrekord University of Medical Sciences, and it is the referral center of the Obstetrics and Gynecology Clinic. The study population was selected among the patients hospitalized in Hajar Hospital in Shahrekord whose pregnancies were terminated because of severe preeclampsia. First, 83 patients were studied regarding to their inclusion criteria and their consent to enter to the study, but 20 of them did not have one or more of the inclusion criteria. Three others refused to consent to enter the study. A total of 60 women who consented to enter the study and signed a consent form were enrolled. The duration of study was short, and no one who wanted to be in the study was excluded. Interventions took place at three and 24 hours after hospitalization, and there were no losses of participants during the study; thus, all of the women were analyzed (Figure 1).

\subsection{Selection criteria}

\subsubsection{Inclusion criteria}

The inclusion criteria for participation in this study were: 1) The mother's age was between 20 and 30;2) the BMIs of the mothers were between 20 and 24;3) gestational age was between 35 and 42 weeks;4) The patient's blood pressure before the termination of her pregnancy was greater than 140/90 $\mathrm{mm} \mathrm{Hg}$; 5)The presence of proteinuria in random urine sample before the termination of the pregnancy; 6) Absence of a history of hypertension, diabetes, kidney disease, collagen vascular disease, and other cardiovascular diseases; 7) Nulliparous mothers and abortion or no previous pregnancy; 8) No smoking or alcohol consumption history in the mother; 9) Singleton not molar pregnancies; 10) Termination of pregnancy is indicated for patients with severe preeclampsia; 11) Patient satisfaction with participating in the study and the consent form was signed; and 12)Lack of preeclampsia before inclusion in the study.

\subsubsection{Exclusion criteria}

The followings were set as the exclusion criteria of the study: 1) The lack of consent to participate in the study and 2) Patinets who were suffering from eclampsia. 


\subsection{Interventions}

The basic information of the patients in the study was recorded in their related forms. The patient's age was calculated based on year of birth and was recorded in form. The patient's weight was measured in kg using an analog scale and recorded. The patient's height was measured in meters by a tape measure, and the patient's BMI was measured based as kilograms per square meter $\left(\mathrm{kg} / \mathrm{m}^{2}\right)$. The patient's type of delivery (vaginal or cesarean) was extracted from the patient's files and was recorded in the related form of each patient. The birth weights of the babies were measured using an infant measuring device, and they were recorded in the related form of each patient. In the baseline, survey results for each patient, including their systolic and diastolic blood pressure, were extracted from their files, and the initial platelet counts were recorded in their related forms. In both the patients group and the control group, the patients received routine, severe preeclampsia treatments, including magnesium sulfate and hydralazine intake if their blood pressures were greater than 110/160. In the patients group, three hours after the termination of pregnancy (3 hours after birth), $70 \mathrm{mg}$ of Silymarin were administered orally, and this was repeated 24 hours later. In the control group, placebo was administered at the same hours (at three hours and then 24 hours later). Twelve, 36, and 60 hours after drug administration, platelet counts were repeated, and the results were recorded in the checklist of each patient.

\subsection{Outcomes}

The primary outcomes of our analyses were the baseline rate of age, BMI, gestational age, Systolic blood pressure $(\mathrm{mmHg})$, diastolic blood pressure $(\mathrm{mmHg})$, average of natal weight at birth $(\mathrm{g})$, platelet count ( $\mathrm{mm} 3 \mathrm{of}$ blood) of the two groups. Also, the secondary outcomes from the analyses were the platelet count at 12, 36, and 60 hours postintervention and the patients' satisfaction with the drug in terms of convenience of use.

\subsection{Sample size}

The sample size was calculated to be 60 subjects. This sample size was calculated based on the results of previous studies (39) by assuming the test power of $80 \%$ and a confidence level of $95 \%$ and using the following formula:

$\mathrm{n}=\left(\mathrm{Z}_{1-\alpha / 2}+\mathrm{Z}_{1-\beta}\right)^{2}\left[\mathrm{P}_{1}\left(1-\mathrm{P}_{1}\right)+\mathrm{P}_{2}\left(1-\mathrm{P}_{2}\right)\right] /\left(\mathrm{P}_{1}-\mathrm{P}_{2}\right)^{2}$

where:

$\mathrm{n}=$ Sample size

$Z_{1-\alpha / 2}=1.96$ when $\alpha=5 \%$ for two-sided test

$\mathrm{Z}_{1-\beta}=0.842$ when $\beta=20 \%$ (test power $=80 \%$ )

$\mathrm{P}=$ Probability of the main outcome

\subsection{Randomization and blinding}

In this clinical trial, stratified randomization was used with the baseline of the termination of pregnancy and diagnosis of severe preeclamsia. After examination of the inclusion criteria, 60 patients entered the study and were divided into two groups, a patients group and a control group. The patients were assigned randomly to the two groups and the priority of reference to clinic to receive either oral silymarin or placebo. Both drugs were of the same size and color. Randomization was done by one of the researchers, who did not have a role in the treatment of the participants. The allocation concealment was done by the researcher who was responsible for the randomization. After being allocated randomly to the groups, all participants were referred to the Hospital's pharmacy to get their drugs. Patients were matched in the two groups for age, BMI, and initial hepatic and platelet enzyme. Also, the outcomes of the study were recorded by the first-year Obstetrics \& Gynecology residents and midwives of the delivery ward who made no other contribution to the study.

\subsection{Statistical methods}

The two groups were compared in terms of age, BMI, baby's birth weight, gestational age at the time of termination of pregnancy, and platelet count, and the data were analyzed using SPSS version 22 (IBMC Corp., Armonk, NY, USA). We used the independent-samples t-test, and ANOVA to analyze the primary and secondary outcomes. The two-tail value of $\mathrm{p}<0.05$ was considered as statistically significant.

\subsection{Research ethics}

The proposal for this thesis research was presented to the Ethics Committee of Shahrekord University of Medical Sciences after its scientific approval by the Obstetrics and Gynecology Department. The Ethics Committee approved the study with the number 92-10-13 on March 15, 2014. The authors confirm that all ongoing and related trials for this drug/intervention are registered. The participants were informed about the objective and nature of the study, and each participant provided her written consent in her native language (Persian) prior to the study. Also, we were 
committed to keeping all of the participants' information confidential. This study also was registered in the Iranian Registry of Clinical Trials with the ID: 201509042388/N1.

\section{Results}

This study included two groups of 30 women with severe preeclampsia who were having their pregnancies terminated. Their characteristics including age, body mass index, gestational age, systolic and diastolic blood pressure platelet at baseline are given in Table 1. The mean age of the participants in the two groups were $25.5 \pm 2.83$ in the case group and $25.4 \pm 3.04$ in the control group $(\mathrm{p}<0.05)$. The mean BMI was $23.6 \pm 0.59$ in the case group and $23.5 \pm 0.54$ in the control group $(\mathrm{p}<0.05)$. The mean gestational age at the time of termination of pregnancy was $36.9 \pm 1.4$ weeks in the case group and $37 \pm 1.5$ in the control group. Mean of platelet count at the baseline was $126 \pm 58.4\left(10^{3}\right)$ in the case group and $122.4 \pm 42.6\left(10^{3}\right)$ in the control group $(\mathrm{p}<0.05)$ (Table 1). The platelet counts at baseline, 12, 36, and $60 \mathrm{~h}$ later were measured, and the results are shown in Table 2 . The mean of platelet count $12 \mathrm{~h}$ after intervention was $126 \pm 58.4\left(10^{3}\right)$ in the case group and $122.4 \pm 42.6\left(10^{3}\right)$ in the control group $(\mathrm{p}<0.05)$. The mean of platelet count $36 \mathrm{~h}$ after intervention was $131.9 \pm 37.5\left(10^{3}\right)$ in the case group and $130.3 \pm 34.9\left(10^{3}\right)$ in the control group $(\mathrm{p}<0.05)$, i.e., there was no statistically significant difference. The mean of platelet count $60 \mathrm{~h}$ after intervention was $140.5 \pm 34.7\left(10^{3}\right)$ in the case group and $139.1 \pm 32.4\left(10^{3}\right)$ in the control group $(\mathrm{p}<0.05)$, which indicates that no statistically significant difference was observed. Blood pressure mean during the study (systolic blood pressure greater than or equal to $160 \mathrm{~mm} \mathrm{Hg}$ and diastolic blood pressure greater than or equal to $110 \mathrm{~mm} \mathrm{Hg}$ ) was $2.1 \pm 0.7$ in the intervention group and $2.4 \pm 0.5$ in the control group, i.e., there was no statistically significant difference between the two groups ( $\mathrm{p} \geq 0.05$ ) (Table 2).

Table 1. Initial variables in study of the participants

\begin{tabular}{|l|l|l|c|}
\hline \multirow{2}{*}{ Variable } & Patients & Control & p-value \\
\cline { 2 - 4 } & Mean \pm SD & Mean \pm SD & \\
\hline Age $($ year $)$ & $25.5 \pm 2.83$ & $25.5 \pm 2.83$ & $>0.05$ \\
\hline BMI $\left(\mathrm{kg} / \mathrm{m}^{2}\right)$ & $23.6 \pm 0.59$ & $23.5 \pm 0.54$ & $>0.05$ \\
\hline Gestational age & $36.9 \pm 1.4$ & $37 \pm 1.5$ & $>0.05$ \\
\hline Systolic blood pressure $(\mathrm{mmHg})$ & $163.8 \pm 1.2$ & $163.4 \pm 4.3$ & $>0.05$ \\
\hline Diastolic blood pressure $(\mathrm{mmHg})$ & $99.1 \pm 5.7$ & $96.4 \pm 5.4$ & $>0.05$ \\
\hline Average of natal weight at birth $(\mathrm{g})$ & $3129.8 \pm 51.6$ & $3167.7 \pm 5.8$ & $>0.05$ \\
\hline Platelet count at baseline $\left(\mathrm{mm}^{3}\right.$ of blood) & $126 \pm 58.4\left(10^{3}\right)$ & $122.4 \pm 42.6\left(10^{3}\right)$ & $>0.05$ \\
\hline
\end{tabular}

Table 2. Platelet count during the study

\begin{tabular}{|l|l|l|l|c|}
\hline \multicolumn{2}{|l|}{ Variables } & Patient group & Control group & p-value \\
\cline { 3 - 5 } \multicolumn{2}{|c|}{} & Mean \pm SD & Mean \pm SD & \\
\hline Platelet count $\left(\mathrm{mm}^{3}\right)$ & At baseline & $126 \pm 58.4\left(10^{3}\right)$ & $122.4 \pm 42.6\left(10^{3}\right)$ & $>0.05$ \\
\cline { 2 - 5 } & After 12 hours & $121.5 \pm 44.2\left(10^{3}\right)$ & $122.1 \pm 42.6\left(10^{3}\right)$ & $>0.05$ \\
\cline { 2 - 5 } & After 36 hours & $131.9 \pm 37.5\left(10^{3}\right)$ & $130.3 \pm 34.9\left(10^{3}\right)$ & $>0.05$ \\
\cline { 2 - 6 } & After 60 hours & $140.5 \pm 34.7\left(10^{3}\right)$ & $139.1 \pm 32.4\left(10^{3}\right)$ & $>0.05$ \\
\hline
\end{tabular}

\section{Discussion}

Presently, there is a limited number of treatments for severe preeclampsia, and several studies have investigated the effect of antioxidant factors in the prevention and treatment of preeclampsia. The aim of this study was to evaluate the effect of Silymarin on platelet abnormalities in severe preeclampsia. Considering the intervention and control groups, there was no statistical difference between the two groups in terms of the ages of the participants in the study. Also, there was no statistical difference between the two groups in terms of BMI $(p>0.05)$. According to these patients, it seems that the intervention and control groups were matched properly for age and BMI, and the effects of confounding factors were minimized in this study. In the two groups in terms of variables of birth weight and gestational age at the time of termination of pregnancy, there were no significant differences between the two groups $(p>0.05)$. Regarding the number of platelets during the study, there was no statistically significant difference between two groups in terms of platelet counts at baseline and at different times during the study. According to the comparative procedure of platelet count between the two groups and the lack of any statistically significant difference in the baseline, it seems that the intervention in this study had no effect on platelet count in patients with severe preeclampsia. Figure 1 shows that platelet count changes were the same in the intervention and control groups. 


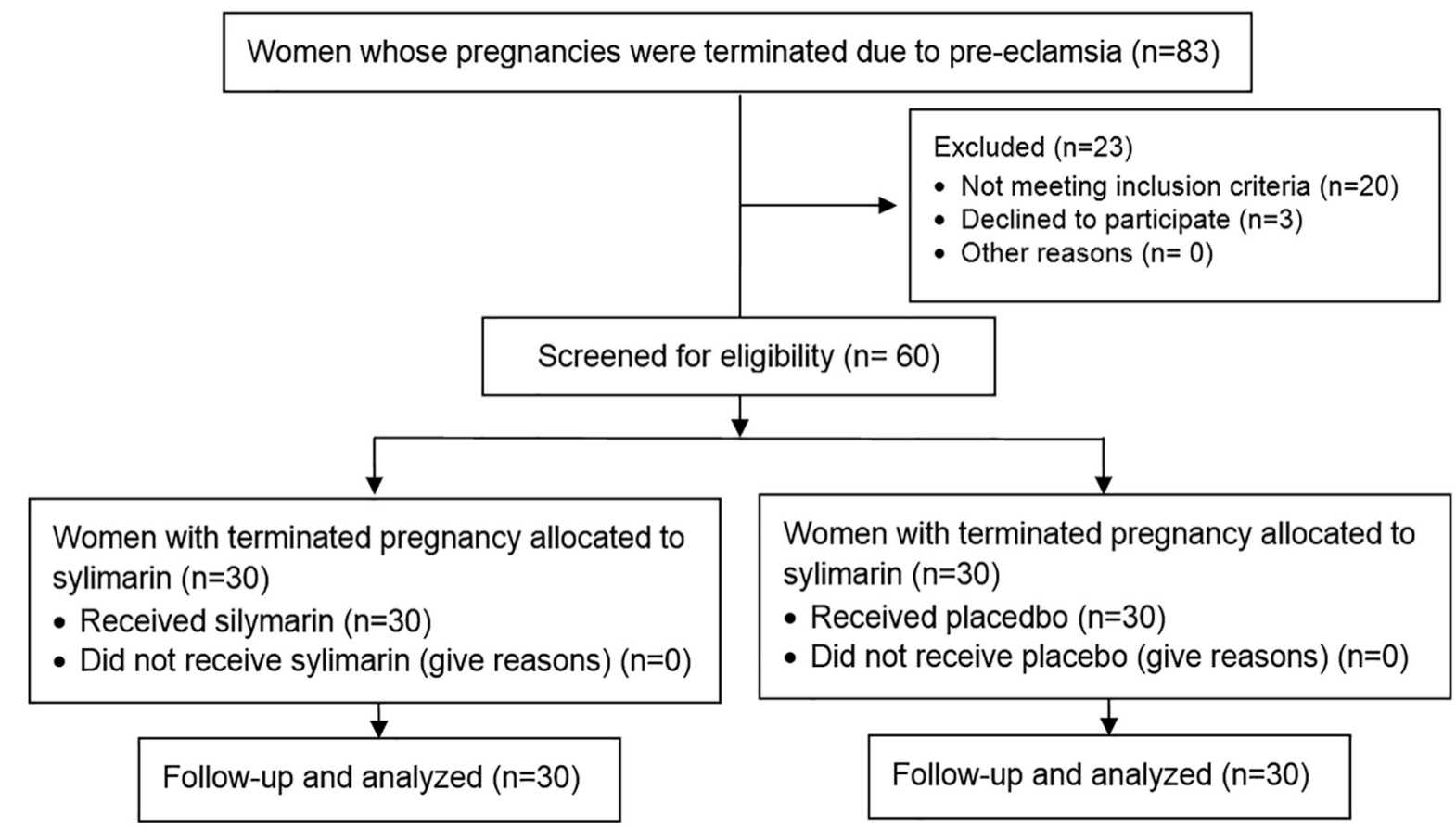

Figure 1. CONSORT flow diagram of the trial

In a study in Ireland, the impact of silymarin on inflammatory cytokines produced by mononuclear cells in preeclampsia was investigated. In this study, it was observed that silymarin reduces the secretion of IL1 $\beta$ and tumor necrosis factor alpha by Peripheral Blood Mononuclear cells (PBMC) in patients with preeclampsia, and it appeared that silymarin exerted antioxidant effects in preeclampsia through these inflammatory mediators (7). In comparing the results of this study that evaluated the performance of silymarin at the cellular level with our study, it was observed in our study that the antioxidant effects of silymarin were not observed and silymarin did not improve platelet disorders in preeclampsia. However, in the aforementioned study, it was noted that the impact of silymarin was investigated at the cellular level and with a biochemical approach, while, in our study, a clinical approach to preeclampsia was emphasized. In a study, the protective effect of silymarin in pregnant wishar rats with preeclampsia was investigated. In that study, $100 \mathrm{mg} / \mathrm{kg}$ of silymarin were administered daily to rats with preeclampsia, and, at the end of a week's treatment, blood pressure, proteinuria, platelet count, liver histopathology, and pregnancy outcomes were evaluated. In the group treated with silymarin, a decrease in blood pressure, proteinuria, and tumor necrosis factor-alpha and interferon-gamma and IL1 $\beta$ and higher platelet counts and improvement in pregnancy outcome was quite obvious and significant.

In the group that was not treated with silymarin, liver histopathological lesions in the periportal region, including mononuclear infiltration, inflammation and thickening of the tunica muscles in the arterial, was quite visible, and these changes were less in the group receiving silymarin. In this study, no adverse effects were observed for the consumption of silymarin in the mother of the fetus (8). In comparing this study that was conducted on pregnant wishar rats with preeclampsia with our study, it was observed that silymarin was effective in reducing blood pressure and increasing the platelet count, but these effects were not observed in our study. This difference could be due to higher doses of silymarin $(100 \mathrm{mg} / \mathrm{kg} / \mathrm{day})$ in the aforementioned study.In this study, the positive impacts of silymarin on the histopathology of the liver, liver enzymes, and proteinuria were observed, but these effects were not evaluated in our study.In one study, the protective effect of silymarin against the effects of intrauterine exposure to alcohol in Sprague-Dawley rats was investigated. In this study, in the groups receiving silymarin, less social intelligence and more anatomical problems were reported (9), and that was consistent with the results of our study. In another study, the effect of silymarin in reducing the symptoms of alcohol syndrome in children with an alcoholic mother was investigated. In this study, the use of silymarin led to higher IQ and weight in babies and no harmful effect was caused by the use of silymarin during pregnancy (10); that is in line with thefindings of our study. It seems that, in the case of confirming safety of silymarin during pregnancy, further studies are needed to approve the efficacy or lack of efficacy of the drug in the above cases. Further studies should be conducted to determine the impact of silymarin before termination of pregnancy in cases of preeclampsia in lengthening the gestational period. 
Also, to ensure the safety of silymarin, studies with higher doses of silymarin should be designed and conducted. In one study, the effect of silymarin in reducing jaundice was investigated, and it was found that silymarin increased the speed of lowering of bilirubin in infantile jaundice. In cases where liver function tests were disrupted, silymarin accelerated their improvement (11). It seems that confirming the safety of silymarin in infants can facilitate its use during the postpartum period and remove the concerns about the potential impacts of the drug in breast milk. One study evaluated the effects of silymarin on thrombophilia in pregnancy and while taking contraceptives pills, and liver enzyme abnormalities were studied. In this study, silymarin at a dose of $50 \mathrm{mg}$ daily for three months was prescribed for patients, and it was observed that silymarin had no effect in reducing the amount of thrombophilia, but it did lower the incidence of hepatic failure (12). In comparing the result of this study with that of our study, it was observed that, in the above-mentioned study, the silymarin dose was $50 \mathrm{mg}$ daily for three months, while in our study, the patients received only two doses of $70 \mathrm{mg}$ of silymarin within 24 hours after delivery. It seems that further studies are needed to better determine the effective and safe dosages of silymarin.

\section{Limitations of study}

In this study, maternal age and gestational age were confounding factors that by matching the intervention and control groups in the study, the confounding effect were decreased. The parity of patient was another confounding factor in the risk of preeclampsia, and to remove the confounding effect of this factor, only nulliparous women was included in the study. Given the presence of people with BMIs of 20 to 24 in this study, the errors caused by differences in body mass index were minimized.

\section{Conclusions}

In this study, the direct effect of silymarin as an antioxidant on the treatment of one of the complications of preeclampsia, i.e., thrombocytopenia, was discussed. It was shown that silymarin did not produce significant changes in the number of platelets. The results of this study also indicated that, although oxidative factors are involved in the incidence of complications of preeclampsia, e.g., thrombocytopenia, the mere use of an oxidative agent cannot contribute to alleviate this effect, and this indicated that it is likely that other factors are involved in the pathogenesis of this disease. Thus, it is recommended that further studies be conducted to prove the beneficial effects of this drug in the treatment of preeclampsia.

\section{Acknowledgments:}

This is part of an MD thesis dissertation supported with project code 2006 by the Deputy of Research and Technology at Shahrekord University of Medical Sciences. We appreciate the patients and members of the hospital staff for their cooperation.

\section{Conflict of Interest:}

There is no conflict of interest to be declared.

\section{Authors' contributions:}

Both authors contributed to this project and article equally. Both authors read and approved the final manuscript.

\section{References:}

1) Gary Cunningham F, Jodi S, Kenneth J, Barbara L, Steven L, Jeanne S, et al. Williams Obstetrics. 24th ed. Newyork: McGraw Hill. 2014; 728-80.

2) Zhang C, Williams MA, King IB, Dashow EE, Sorensen TK, Frederio IO, et al. Vitamin C and the risk of preeclampsia resaits from dietary questionnaire and plasma assay. Epidemiology. 2002; 13(4): 409-16. doi: 10.1097/00001648-200207000-00008. PMID: 12094095.

3) Gilbert E, Harman J. Manual of high risk pregnancy and delivery. 2th ed. St Louis: Mosby. $2003 ; 445-62$.

4) Conlin PR, Chow D, Miller ER, Svetkey LP, Lin PH, Harsha DW, et al. The effect of dietary patterns on blood pressure control in hypertensive patients: Results from the dietary approaches to stop hypertention (Dash) trial. Am J hypertens. 2000; 13(9): 949-55. doi: 10.1016/S0895-7061(99)00284-8. PMID: 10981543.

5) Langmead L, Rampton DS. Review article: Herbal treatment in gastrointestinal and liver disease. Aliment Pharmacol Ther. 2001; 15(9): 1239-52. doi: 10.1046/j.1365-2036.2001.01053.x. PMID: 11552894.

6) Abenavoli L, Capasso R, Milic N, Capasso F. Milk thistle in liver disease: past, present, future. Phtother Res. 2010; 24(10): 1423-32. doi: 10.1002/ptr.3207. PMID: 20564545. 
7) Giorgi VS, Peracoli MT, Peracoli JC, Witkin SS, Bannwart-Castro CF. Silibin modulates the NF-kb pathway and proinflammatory cytokine production by mononuclear cells from preeclamptic women. J Reprod Immunol. 2012; 95(1-2): 67-72. doi: 10.1016/j.jri.2012.06.004. PMID: 22871551.

8) Souza CO, Peracoli MT, Weel IC, Bannwart CF, Romao M, Nakaira-Takahagi E, et al. Hepatoprotective and anti-inflammatory effects of silibin on experimental preeclampsia induced by L-NAME in rats. Life sci. 2012; 91(5-6): 159-65. doi:10.1016/j.1fs.2012.06.036. PMID: 22781706.

9) Reid C, Edwards J, Wang M, Manybeads Y, Mike L, Martinez N, et al. Prevention by a silymarin phospholipid compound of ethanol induced social learning deficits in rats. Planta Med. 1999; 65(5): $421-4$. doi: 10.1055/s-1999-14085. PMID: 10418328 .

10) La Grange L, Wang M, Watkins R, Ortiz D, Sanchez ME, Konst J, et al. Protective effects of the flavonoid mixture silymarin on fetal brain and liver. J Ethnopharm. 2009; 6(5): 53-61. doi:10.1016/S03788741(98)00144-5. PMID: 10418328.

11) Kassem LM, Abdelrahim MEA, Naguib HF. Investigating the efficacy and safety of silymarin in management of hyperbilirubinemia in neonatal jaundice. Med Sci. 2013; 2(2): 575-90. doi: 10.5455/medscience.2013.02.8057.

12) Martines G, Pira M, Copponi V, Cagnetta G. silymarin in pregnancy and during hormonal contraceptive treatment. Arch sci Med (Torino). 1979; 136(3): 443-54. PMID: 549550. 